\title{
Integrated Balancing and Congestion Management under Forecast Uncertainty
}

\section{Conference Paper}

\section{Author(s):}

Roald, Line; Krause, Thilo; Andersson, Göran

Publication date:

2016

Permanent link:

https://doi.org/10.3929/ethz-a-010607953

Rights / license:

In Copyright - Non-Commercial Use Permitted

Originally published in:

https://doi.org/10.1109/ENERGYCON.2016.7513980 


\title{
Integrated Balancing and Congestion Management under Forecast Uncertainty
}

\author{
Line Roald, Student Member, IEEE, Thilo Krause, Member, IEEE, Göran Andersson, Fellow, IEEE \\ Power Systems Laboratory, Department of Electrical Engineering, ETH Zurich, Switzerland \\ Email: \{roald | krause | andersson\}@eeh.ee.ethz.ch
}

\begin{abstract}
Forecast uncertainty related to the power production from renewables frequently leads to significant changes between the scheduled and the actual power injections. Larger and more frequent fluctuations in the power production require new approaches to handle congestion and ensure system security. In this paper, we investigate how different strategies for reserve activation influence the cost of integrating uncertain in-feeds. In particular, we assess under which conditions it is beneficial for the TSOs to balance across a large region and when deviations should be handled close to where they originate. Together with the mathematical formulation of the corresponding OPF model, which involves chance constraints to account for uncertainty, we show how the chance constraints can be reformulated to form a tractable optimization problem. We demonstrate the feasibility of our approach on the IEEE 118 bus system. The case study illustrates in particular the influence of the different balancing policies on the overall system cost as well as on the utilisation of the transmission system. Comparisons are made between the deterministic and the probabilistic approaches in conjunction with the modelled balancing and congestion management schemes. The results show how accounting for congestion when activating reserves can help reducing the overall cost of handling uncertainty.
\end{abstract}

Index Terms-Renewable integration, forecast uncertainty, congestion management, chance constrained optimal power flow

\section{INTRODUCTION}

With higher shares of electricity generation from renewable energy sources, transmission system operators (TSOs) experience more uncertainty in power system operation [1]. In particular, forecast uncertainty related to the power production from renewables frequently leads to significant changes between the power plant schedules and the actual power injections. For the system operator, increased uncertainty has several implications. First, larger and more frequent fluctuations in the power production lead to an increased need for reserve capacity and reserve activation to keep the power balance. Second, changes in the power injections impact the power flows in the system, and hence new approaches to handle congestion are required.

Since fluctuations in the power production from renewable sources are not perfectly correlated, deviations across a large

This research work described in this paper has been carried out within the scope of the project "Innovative tools for future coordinated and stable operation of the pan-European electricity transmission system (UMBRELLA)", supported under the 7th Framework Programme of the European Union, grant agreement 282775 . geographical area may partially cancel out. The total power mismatch thus tends to be smaller for a larger area. To achieve power system balance with minimum reserve activation, the balancing area should be as large as possible. This effect has lead to the implementation of inter-TSO balancing schemes such as imbalance netting [2], [3], where active power imbalances between TSOs are netted (i.e., cancelled out against each other) before the control reserves are activated. As an example, the European initiative International Grid Control Cooperation (IGCC) started in 2011 with imbalance netting between the four German and the Danish TSOs, and has later gradually expanded to include 10 TSOs in 7 countries [4]. By January, IGCC had avoided activation of more than $3.8 \mathrm{TWh}$ worth of control reserves [4].

However, while larger balancing areas reduce the amount of reserve activation, a power mismatch might be balanced far away from where it occurred. This leads to more significant changes in the power flows, and complicates congestion management. For example, an unscheduled increase in the wind power close to the north of Europe and subsequent down-regulation by a power plant in the south might induce additional loop flows and overloads on transmission lines transporting power between the two different parts of the grid. If not planned appropriately, imbalance netting might thus necessitate additional (costly) redispatch measures, which undermine part of the the cost saving reasoning.

The TSO thus faces a trade-off: While balancing across a large region, i.e. global balancing, reduces the requirement on reserves, it might increase the cost of congestion management. An approach where the deviations are balanced close to where they originated, i.e. local balancing, simplifies congestion management, but requires more reserves. The purpose of this paper is to investigate under which conditions each of the two approaches (local or global balancing) are cost-effective.

The importance of accounting for grid constraints in interzonal balancing operations was also investigated in, e.g., [5], but with a focus on market integration and only a limited set of transmission constraints. Here, we focus on security aspects in transmission system operational planning, and incorporate a more detailed model of both the transmission grid, the forecast uncertainty and the available reserves. To assess the impact of forecast uncertainty and reserve activation, we formulate a chance constrained optimal power flow (CC-OPF) along the lines of [6], [7]. The CC-OPF ensures that the system will remain secure with a high probability, despite the 
forecast uncertainty. Specifically, we show how the chance constraints ensure a low probability of overloads by reserving appropriate capacity on transmission lines that are influenced by uncertainties and reserve activation.

The contributions of this paper are threefold. First, we extend previous CC-OPF models [6]-[8] to include a local balancing policy which accounts for the location of the deviations. Second, we qualitatively discuss how the new formulation relates to two global balancing policies, where i) the generators provide reserves based on predefined participation factors (based on [6]) and ii) the participation factors are optimized (based on [7]). Third, we compare the three balancing policies in a case study for the IEEE 118 bus system with 91 uncertain in-feeds. We assess and explain the differences in cost and congestion between the different balancing approaches, and show how the local balancing policy combines the benefits of imbalance netting and congestion management.

The remainder of the paper is structured as follows. Section II details the CC-OPF formulation for the three different balancing policies. It also describes how the chance constraints are reformulated to form a tractable optimization problem, and discusses the problem complexity for each policy. Section III presents the case study set-up and the results. Section IV concludes the paper.

\section{OPTIMAL POWER FLOW FORMULATION}

For a quantitative comparison of different balancing and congestion management approaches, we extend the CC-OPF proposed in [6], [7] to allow for local balancing of wind power deviations. The formulation is based on a DC power flow approximation. In this section, we both review previous formulations and introduce new modelling aspects.

We denote vectors by lower case letters $p, \omega$. The components of the vectors are denoted by using subscripts, i.e, the $i$ th component of $p$ is denoted by $p_{i}$. Matrices are denoted by upper/lower bold case letters, $\boldsymbol{\alpha}, \mathbf{M}$, and $\boldsymbol{\alpha}_{(i, \cdot)}, \boldsymbol{\alpha}_{(\cdot, i)}$ denote the $i^{\text {th }}$ row and column of $\boldsymbol{\alpha}$, respectively. Index $i$ refers to generators, while index $i j$ refers to lines.

\section{A. System modelling}

We consider a power system where $\mathcal{N}$ is the set of nodes and $\mathcal{L}$ is the set of lines. The number of nodes and lines are given by $|\mathcal{N}|=m$ and $|\mathcal{L}|=l$, respectively. The set of nodes with uncertain demand or production of energy is given by $\mathcal{U} \subseteq \mathcal{N}$. The fluctuations in the demand or production at any given node can result from various sources, such as load fluctuations, forecast errors for wind or PV or intraday electricity trading. The set of controllable, conventional generators is denoted by $\mathcal{G} \subseteq \mathcal{N}$. To simplify the notation, we assume that there is one conventional generator $p_{i}$, one composite uncertainty source $u_{i}$ and one demand $d_{i}$ per node, such that $|\mathcal{G}|=|\mathcal{U}|=|\mathcal{N}|=m$. Nodes without generation or load can be handled by setting the respective entries to zero, and nodes with multiple entries can be handled through a summation. To focus on the handling of fluctuations, the formulation presented here omits $\mathrm{N}-1$ security constraints. These can easily be included within the presented formulation, although at an increase in computational complexity.
1) Uncertainty sources: The uncertainty sources $u \in \mathbb{R}^{m}$ are modeled as the sum of the forecasted production of active power (e.g., from wind or solar PV) $\mu \in \mathbb{R}^{m}$ and a zero mean fluctuating component $\omega \in \mathbb{R}^{m}$ :

$$
u=\mu+\omega .
$$

The fluctuating component $\omega$ can contain any source of uncertainty, such as forecast errors for renewables, load uncertainty or schedule changes due to intra-day trading.

2) Power balance, generation control and reserves: The controllable generators are required to produce power such that they ensure power balance at all times. At the nominal operating point (with $\omega=0$ ), the total power balance in the system is given by the following equality constraint:

$$
\sum_{i \in \mathcal{N}} p_{i}-d_{i}+\mu_{i}=0 .
$$

To balance fluctuations in energy demand or production, we assume that the generators adjust their in-feeds according to an automatic generation control (AGC) signal. The amount of requested up- or down-regulation is proportional to the fluctuation, but with opposite sign, leading to an affine control policy as in [6]-[8]. Modelling the generator in-feeds in this way has several advantages: First, we can treat the fluctuations as continuous variables (as opposed to a representation through a finite number of scenarios). Second, it provides an affine control policy which can be implemented by the TSO. Third, it allows us to account for uncertainty in operational planning without compromising computational tractability, as will become apparent in subsequent sections.

The total available up- and down-reserve capacities that can be used for balancing are given by $r^{+}, r^{-} \in \mathbb{R}^{m}$. Since European reserve markets are typically cleared at a weekly or daily basis, we assume that the total available capacities for reserve provision from each generator, modelled by $r^{+}, r^{-}$, are fixed parameters in the current formulation. The reserve procurement is assumed to be conservative (i.e., there is sufficient reserves to cover relevant fluctuations), and the reserves are assumed to be symmetric, such that $r^{+}=r^{-}$. Further, we assume that generators have passed prequalification tests which ensure that their ramping capabilities enable sufficiently fast activation of reserves. Note that the formulation can easily be extended to optimize the reserve procurement by introducing $r^{+}, r^{-}$as optimization variables.

In the following, we will consider three different ways of defining the balancing policy. The two first policies are based on global balancing, where the generators only react to the overall power deviation $\Omega \doteq \sum_{i \in \mathcal{U}} \omega$, while the last policy is based on local balancing, where the generators react directly to the local deviations $\omega$.

Policy I - Global balancing with predefined policy: As in [6], [8], the contribution of balancing energy from each generator is a predetermined input to the optimization problem. The contribution can be chosen in different ways. For this paper, we assume that each generator contributes balancing energy according to its share of total procured reserves, such 
that the actual generator output $\tilde{p}$ is given by

$$
\tilde{p}=p-\alpha_{1} \Omega \quad \text { where } \quad \alpha_{1}=\frac{r^{+}}{\sum_{i \in \mathcal{G}} r_{i}^{+}} \in \mathbb{R}^{m} .
$$

By definition, we have $\sum_{i \in \mathcal{G}} \alpha_{1, i}=1$, which ensures that any given fluctuation $\Omega$ is balanced by the same amount $\sum_{i \in \mathcal{G}} \alpha_{1, i} \Omega=\Omega$. This balancing policy corresponds to the situation where the system operator procures reserves without consideration of where the reserves are located.

Policy II - Optimal global balancing: Based on [7], the contribution of each generator is given by

$$
\tilde{p}=p-\alpha_{2} \Omega,
$$

where the balancing vector $\alpha_{2} \in \mathbb{R}^{m}$ is subject to optimization. To ensure power balance during fluctuations and activation of only up or down reserves, we enforce the following constraints on $\alpha_{2}$ :

$$
\sum_{i \in \mathcal{G}} \alpha_{2, i}=1, \quad \alpha_{2} \geq 0
$$

This balancing policy corresponds to a situation where the TSO is able to decide where the balancing should happen in the system, and can use this ability to influence how reserve activation changes the power flows in the system.

Policy III - Optimal local balancing: For the local balancing case, the generators are reacting to each wind power plant separately. The contribution of each generator is given by

$$
\tilde{p}=p-\boldsymbol{\alpha}_{3} \omega
$$

where $\boldsymbol{\alpha}_{3} \in \mathbb{R}^{m \times m}$ is a matrix describing the individual balancing of each uncertainty source. As for Policy II, the balancing matrix $\boldsymbol{\alpha}_{3}$ is subject to optimization. To enforce power balance during deviations and activation of only up- or down-reserves, we add the following constraints:

$$
\sum_{i \in \mathcal{G}} \boldsymbol{\alpha}_{3(i, j)}=1 \quad \forall j \in \mathcal{U}, \quad \boldsymbol{\alpha}_{3} \geq 0
$$

Here, the first equation ensures that the activation of balancing energy for any individual fluctuation $\omega_{j}$ is equal to the fluctuation itself, i.e., $\sum_{i \in \mathcal{G}} \boldsymbol{\alpha}_{3(i, j)} \omega_{j}=\omega_{j}$. By optimizing the full matrix $\boldsymbol{\alpha}_{3}$, the TSO has more influence on how balancing changes power flows. In particular, the deviations can be balanced locally in parts of the system with significant congestion. On the other hand, the optimization can let the deviations cancel each other, e.g. in a geographically close region without congestion, by choosing balancing vectors $\boldsymbol{\alpha}_{3}(i, \cdot)=\boldsymbol{\alpha}_{3}(j, \cdot)$. Policy III thus allows us to evaluate the trade-off between local and global balancing, and to quantify the benefits of moving from current balancing practice towards a more complex policy based on the location of system reserves.

For the remainder of the paper, we will use the following short-hand notation to express Policies I-III,

$$
\tilde{p}=p-\boldsymbol{\alpha} \omega
$$

where $\boldsymbol{\alpha} \in \mathbb{R}^{m \times m}$ is given by

$$
\begin{aligned}
& \boldsymbol{\alpha}=\left[\begin{array}{llll}
\alpha_{1} & \alpha_{1} & \ldots & \alpha_{1}
\end{array}\right] \\
& \boldsymbol{\alpha}=\left[\begin{array}{llll}
\alpha_{2} & \alpha_{2} & \ldots & \alpha_{2}
\end{array}\right] \\
& \boldsymbol{\alpha}=\boldsymbol{\alpha}_{3} \\
& \text { for Policy I, } \\
& \text { for Policy II, } \\
& \text { for Policy III, }
\end{aligned}
$$

in the three different cases. Note that $\alpha_{1}$ and $\alpha_{2}$ are special cases of $\boldsymbol{\alpha}_{\mathbf{3}}$.

3) Power flow modelling: The power flows $p_{i j}$ on each line are computed according to the DC approximation

$$
p_{i j}=\mathbf{M}_{(i j, \cdot)}(p-\boldsymbol{\alpha} \omega+\mu+\omega-d), \forall_{i j \in \mathcal{E}} .
$$

The matrix $\mathbf{M} \in \mathbb{R}^{n \times m}$ is the matrix of Power Transfer Distribution Factors (PTDFs). It relates the line flows to the nodal power injections, which are expressed as the sum of generation $p-\boldsymbol{\alpha} \omega$, wind power production $v+\omega$ and demand $-d . \mathbf{M}$ is defined as

$$
\mathbf{M}=B_{f}\left[\begin{array}{cc}
\left(\widetilde{B}_{b u s}\right)^{-1} & \mathbf{0} \\
\mathbf{0} & 0
\end{array}\right]
$$

where $B_{f}$ is the line susceptance matrix and $\widetilde{B}_{b u s}$ the bus susceptance matrix (without the last column and row, corresponding to the slack bus) [9]. $\mathbf{M}_{(i j, \cdot)}$ is the row of $\mathbf{M}$ related to the line $(i j) \in \mathcal{L}$.

\section{B. Optimal Power Flow Formulation}

With the above modelling considerations, the $\mathrm{CC}-\mathrm{OPF}$ is stated as follows:

$$
\begin{aligned}
& \min _{p, \boldsymbol{\alpha}} \sum_{i \in \mathcal{G}} c_{i} p_{i} \\
& \sum_{i \in \mathcal{N}} p_{i}-d_{i}+\mu_{i}=0 \\
& \sum_{i \in \mathcal{G}} \boldsymbol{\alpha}_{(i, j)}=1 \quad \forall{ }_{j \in \mathcal{U}}, \quad \boldsymbol{\alpha} \geq 0 \\
& p+r^{+} \leq p^{\max } \\
& p-r^{-} \geq p^{\min } \\
& \mathbb{P}\left[-\boldsymbol{\alpha}_{(i, \cdot)} \omega \leq r_{i}^{+}\right] \geq 1-\epsilon_{g} \quad \forall_{i \in \mathcal{G}} \\
& \mathbb{P}\left[-\boldsymbol{\alpha}_{(i, \cdot)} \omega \geq r_{i}^{-}\right] \geq 1-\epsilon_{g} \quad \forall_{i \in \mathcal{G}} \\
& \mathbb{P}\left[\mathbf{M}_{(i j, \cdot)}(p-\boldsymbol{\alpha} \omega-d+\mu+\omega) \leq \bar{p}_{i j}\right] \geq 1-\epsilon_{l} \quad \forall_{\{i j\} \in \mathcal{L}} \\
& \mathbb{P}\left[\mathbf{M}_{(i j, \cdot)}(p-\boldsymbol{\alpha} \omega-d+\mu+\omega) \geq-\bar{p}_{i j}\right] \leq 1-\epsilon_{l} \quad \forall_{\{i j\} \in \mathcal{L}}
\end{aligned}
$$

The objective (11) is to minimize generation cost, where $c$ contains costs, i.e. bids, from the generators. Eq. (13) reflects the power balance during nominal operation, while (14) enforces the power balance during fluctuations and a positive $\alpha$. Constraints (15) - (16) ensure that the scheduled generation $p$, in combination with the reserve capacities $r^{+}, r^{-}$, remain within the upper and lower generation limits $p^{\max }, p^{\min }$. Eq. (17), (18) ensure that the generators assigned to provide reserves have sufficient reserve capacity to cover the fluctuations, while (19), (20) ensure that the transmission constraints are not violated. 
Since the generation output and the power flows depend on the fluctuations $\omega$, the reserve constraints (17), (18) and transmission constraints (19), (20) are formulated as separate chance constraints. Each constraint is enforced with an accepted violation probability, $\epsilon_{g}$ for the generator constraints and $\epsilon_{l}$ for the line constraints.

\section{Chance constraint reformulation}

To obtain a tractable optimization problem, the chance constraints must be reformulated into deterministic constraints. There are different ways of doing this reformulation, with two main directions being sampling-based approaches [8], [10] or analytical reformulations [6], [7], [11]. Here, we choose to apply an analytical reformulation similar to the ones used in [6], [7], but extend the formulation to account for a full covariance matrix $\Sigma$ of the fluctuations. This allows us to account for correlation between the different fluctuations $\omega$. The constraints are reformulated analytically based on the assumption of normally distributed line flows and generator outputs. Assuming a normal distribution is reasonable in systems where the fluctuations are close to normal (e.g., mainly load uncertainty). However, even when the fluctuations are not normally distributed, the changes in the line flows and generator outputs (which are weighted sums of the fluctuations themselves) might be close to normal, particularly in systems where the number of uncertainty sources is large and not highly correlated. In this case, we can apply arguments similar to the central limit theorem [12].

By applying the properties of the normal distribution, the chance constraints (17) - (20) can be reformulated as secondorder cone (SOC) constraints [11]. We explain the steps in more detail based on the line constraint (19) for a particular line $i j$. We observe that the expression on the left hand side (representing the line flow) is a weighted sum of the entries of the vector $\omega$. Assuming that the $\omega$ follows a multivariate normal distribution, the weighted sum is also normally distributed. Therefore, we first normalize the left hand side of the constraint to a standard normal variable with zero mean and unit variance,

$$
\mathbb{P}\left[\frac{\mathbf{M}_{(i j, \cdot)}(-\boldsymbol{\alpha} \omega+\omega)}{\left\|\mathbf{M}_{(i j, \cdot)}(\boldsymbol{I}-\boldsymbol{\alpha}) \Sigma^{1 / 2}\right\|_{2}} \leq \frac{\bar{p}_{i j}-\mathbf{M}_{(i j, \cdot)}(p-d+\mu)}{\| \mathbf{M}_{(i j, \cdot)}(\boldsymbol{I}-\boldsymbol{\alpha})^{\Sigma^{1 / 2} \|_{2}}}\right] \geq 1-\epsilon
$$

where $\left\|\mathbf{M}_{(i j,)}(\boldsymbol{I}-\boldsymbol{\alpha} \omega) \Sigma^{1 / 2}\right\|_{2}$ represents the standard deviation of the line flow. With the cumulative distribution function $\Phi$ of the standard normal distribution, (21) can now be expressed as

$$
\Phi\left(\frac{\bar{p}_{i j}-\mathbf{M}_{(i j, \cdot)}(p-d+\mu)}{\left\|\mathbf{M}_{(i j, \cdot)}(\boldsymbol{I}-\boldsymbol{\alpha}) \Sigma^{1 / 2}\right\|_{2}}\right) \geq 1-\epsilon .
$$

By inversing the cumulative distribution function, we obtain

$$
\frac{\bar{p}_{i j}-\mathbf{M}_{(i j, \cdot)}(p-d+\mu)}{\left\|\mathbf{M}_{(i j, \cdot)}(\boldsymbol{I}-\boldsymbol{\alpha}) \Sigma^{1 / 2}\right\|_{2}} \geq \Phi^{-} 1(1-\epsilon)
$$

By repeating these steps for the remaining chance constraints and rearranging terms, we obtain the following reformulation for (17) - (20):

$$
\begin{aligned}
& \Phi^{-1}(1-\epsilon)\left\|\boldsymbol{\alpha}_{(\cdot, i)}^{T} \Sigma^{1 / 2}\right\|_{2} \leq r^{+}, \quad \forall\{i\} \in \mathcal{G} \\
& -\Phi^{-1}(1-\epsilon)\left\|\boldsymbol{\alpha}_{(\cdot, i)}^{T} \Sigma^{1 / 2}\right\|_{2} \geq-r^{-}, \quad \forall\{i\} \in \mathcal{G} \\
& \mathbf{M}_{(i j, \cdot)}(p-d+\mu) \leq \bar{p}_{i j}- \\
& \Phi^{-1}(1-\epsilon)\left\|\mathbf{M}_{(i j, \cdot)}(\boldsymbol{I}-\boldsymbol{\alpha}) \Sigma^{1 / 2}\right\|_{2}, \quad \forall_{\{i j\} \in \mathcal{L}} \\
& \mathbf{M}_{(i j, \cdot)}(p-d+\mu) \geq-\bar{p}_{i j}+ \\
& \Phi^{-1}(1-\epsilon)\left\|\mathbf{M}_{(i j, \cdot)}(\boldsymbol{I}-\boldsymbol{\alpha}) \Sigma^{1 / 2}\right\|_{2}, \quad \forall_{\{i j\} \in \mathcal{L}}
\end{aligned}
$$

By looking at the constraints (17) - (20), we observe that a higher value of $\Phi^{-1}(1-\epsilon)$ implies a tightening of the constraint. This is particularly evident for the line flow constraints (26), (27), where the term

$$
U_{i j}=\Phi^{-1}(1-\epsilon)\left\|\mathbf{M}_{(i j, \cdot)}(\boldsymbol{I}-\boldsymbol{\alpha} \omega) \Sigma^{1 / 2}\right\|_{2}
$$

has a direct interpretation as a reduction of the available transmission capacity. This reduction in transmission capacity can be seen as the security margin which is necessary to secure the system against uncertainties, which we will refer to as the uncertainty margin $U_{i j}$.

From the uncertainty margin (28) it is clear how Policies I-III have different effects on congestion. For Policy I, the variables $\boldsymbol{\alpha}$ are predetermined, which implies that (28) is a fixed quantity. For Policy II and III, the values of $\boldsymbol{\alpha}$ can be optimized to reduce the uncertainty margin on congested lines (thus increasing nominal transmission capacity). Policy III has a higher potential to reduce the uncertainty margins than Policy II, since it reacts to the individual fluctuations.

\section{Problem complexity}

For Policy I where $\boldsymbol{\alpha}$ is fixed, the uncertainty margins (28) are known quantities that can be pre-calculated. Moreover, with $\alpha_{1}$ defined according to (3), the reserve constraints (24), (25) will be feasible whenever enough reserves $\sum_{i \in \mathcal{G}} r^{+}=$ $\sum_{i \in \mathcal{G}} r^{-} \leq \Omega$ have been procured, and can thus be omitted from the optimization problem. The CC-OPF with Policy I thus remains a linear program with the same solution complexity as the corresponding deterministic OPF.

For Policy II, the generation constraints (24), (25) can be simplified [13]. We express $\boldsymbol{\alpha}=\alpha \cdot \mathbf{1}_{\{1, m\}}$, such that (24) reduces to the following linear constraint:

$$
\begin{array}{ll}
\alpha_{i} \Phi^{-1}(1-\epsilon)\left\|\mathbf{1}_{\{1, m\}}^{T} \sum^{\Sigma^{1 / 2}}\right\|_{2} \leq r^{+} & \forall_{\{i\} \in \mathcal{G}}, \\
\alpha_{i} \Phi^{-1}(1-\epsilon)\left\|\mathbf{1}_{\{1, m\}}^{T} \Sigma^{1 / 2}\right\|_{2} \leq r^{-} & \forall_{\{i\} \in \mathcal{G}} .
\end{array}
$$

The transmission constraints (19), (20) do however remain SOC constraints increasing the computational complexity. The SOC constraints are however convex, which allows for the design of efficient solution algorithms along the lines of [7].

For Policy III, both generation and transmission constraints (24) - (27) are true SOC constraints. In addition, the solution complexity increases due to the larger number of variables $\boldsymbol{\alpha}_{3}$ compared with $\alpha_{2}$. 


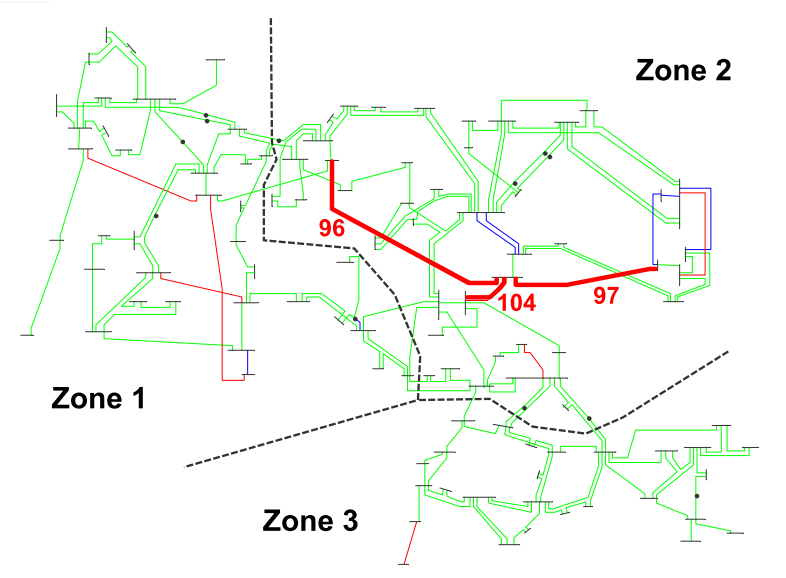

Fig. 1. IEEE 118 bus system with 3 Zones and marking of the lines 96, 97 and 104. The color of the lines reflect the loading in the deterministic OPF solution, with green $<0.9 p_{i j}^{\max }$, blue $0.9-0.99 p_{i j}^{\max }$ and red $>$ $0.99 p_{i j}^{\max }$

\section{CASE STUDY}

In this case study, we investigate how the different balancing policies impacts system operation. We use the IEEE 118 bus test systems as defined in [14], with the following modifications to make the system more congested. The system is divided into three zones, as seen in Fig. 1. The load is increased by a factor of 1.5 in zone 1 and 2, and by a factor of 2 in zone 3 . The transfer capacities of the transmission lines are decreased to $75 \%$ of the original capacity. The generation capacity of all generators is increased by a factor of 3 , and the available reserves capacities $r^{+}, r^{-}$are set to $15 \%$ of the total generation capacity, i.e. $r^{+}=r^{-}=0.15 p^{\max }$.

The system loads are interpreted as a mix between load and renewable energy sources connected at a lower voltage level. Instead of considering that particular wind in-feeds are uncertain, we assume that all 91 loads fluctuate around their forecasted consumption, and that the standard deviation $\sigma$ of each load is equal to $20 \%$ of the forecasted consumption. For simplicity, we further assume that the fluctuations are uncorrelated, such that $\Sigma=\operatorname{diag}(\sigma)$, although the method can handle correlation by introducing off-diagonal elements in $\Sigma$. We choose acceptable violation probabilities $\epsilon_{g}=\epsilon_{l}=0.05$.

The CC-OPF is implemented in MatLab using Yalmip [15] to formulate the SOC constraints, and is solved using CPLEX. We compare the CC-OPF with a deterministic OPF, which corresponds to the CC-OPF with $\omega=0$.

\section{A. Results}

We first compare the nominal cost between the deterministic solution and the three chance-constrained solutions corresponding to Policy I, II and III. Second, we explain the differences in cost by looking at the uncertainty margins of different congested lines. Finally, we discuss how balancing is distributed between generators for Policies I, II and III.

1) Cost of uncertainty: In Fig. 2, the nominal cost of the generation dispatch (i.e., the value of the OPF objective function) is shown for the deterministic solution, as well as for the

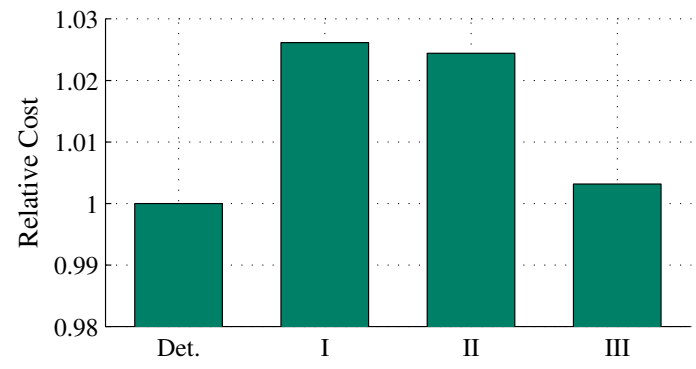

Fig. 2. Cost of optimal power flow solution, normalized by the cost of the deterministic solution. Det.: Deterministic OPF. Policy I: CC-OPF with predetermined, global balancing. Policy II: CC-OPF with optimal, global balancing. Policy III: CC-OPF with optimal, local balancing.

chance constrained problem with the three different policies. All costs are normalized by the cost of the deterministic solution. We observe that all chance constrained solutions lead to a cost increase compared with the deterministic solution. With Policy I, the increase amounts to $+2.5 \%$, while Policy II is slightly less expensive with $+2.3 \%$. Policy III only results in higher costs of $+0.2 \%$.

While the nominal cost of the generation dispatch increases when accounting for uncertainty, the solution obtained through either of the chance constrained approaches is more secure and will lead to significantly fewer violations of generation and transmission constraints in real time. The difference in the cost increase between Policies I-III show that the cost of integrating uncertainty in power system operations significantly varies dependent on different balancing strategies. In the following, we explain the reasons for the difference in cost.

2) Uncertainty margins: As discussed in Section II-C, accounting for uncertainty leads to a reduction in transmission capacity. Being able to influence the uncertainty margins through the choice of $\alpha_{2}$ (with Policy II) or $\boldsymbol{\alpha}_{3}$ (in Policy III) is however useful to reduce the uncertainty margins on congested lines. Examples of such lines are line 96, 97 and 104 in Fig. 1. The uncertainty margins $U_{i j}$ for these lines are shown in Fig. 3. We observe that compared with the predefined Policy I, both Policy II and III reduce the necessary uncertainty margins. For Policy III, the uncertainty margins on the critical lines are almost zero.

With smaller uncertainty margins, more transmission capacity is available for transportation of energy from lowcost zones to high-cost zones, which decreases overall cost. The differences in the uncertainty margins explain the cost differences seen in Fig. 2.

3) Control policy: Finally, we assess how the control policy $\boldsymbol{\alpha}$ behaves for the different policies. Fig. 4 shows the value of the different entries of $\alpha_{1}, \alpha_{2}$ (vectors) and $\alpha_{3}$ (matrix). For the global Policies I and II, the balancing is spread relatively evenly across a large number of generators. The largest entries are $\max \left\{\alpha_{1}\right\}=0.05$ and $\max \left\{\alpha_{2}\right\}=0.22$, respectively. For the local Policy III, the majority of any single fluctuation $\omega_{j}$ is typically covered by a single generator. Further, we observe that the uncertain loads located geographically close to each other are typically balanced through the same generators, such 


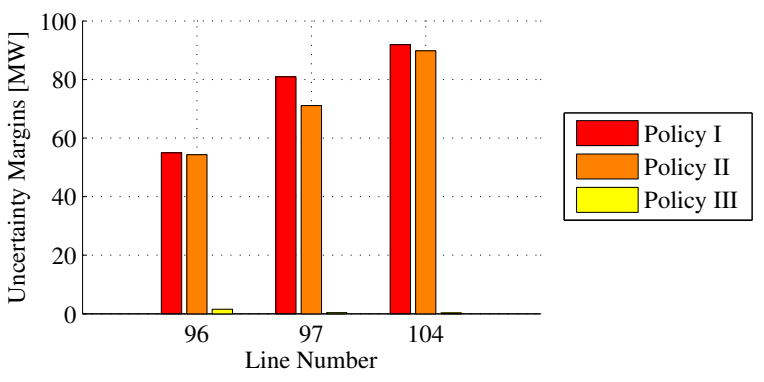

Fig. 3. Uncertainty margins $U_{i j}$ for lines 96,97 and 104 with the three different policies.

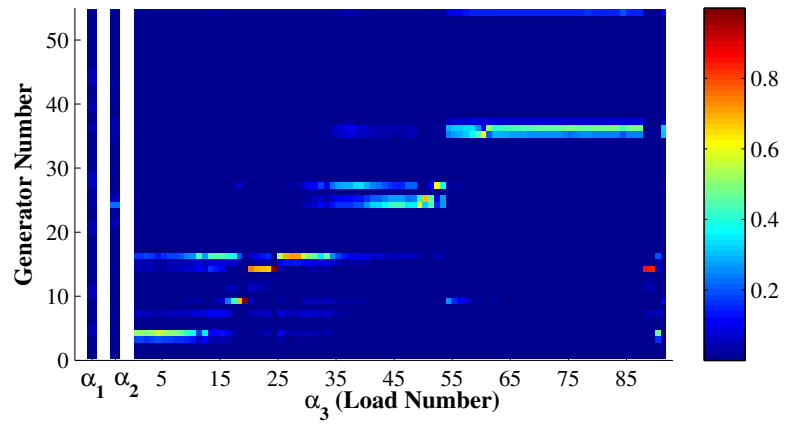

Fig. 4. Value of $\boldsymbol{\alpha}$ for the three different balancing policies. To the left: The balancing vectors $\alpha_{1}, \alpha_{2}$, where the generators react only to the overall power mismatch (one entry per generator). To the right: The balancing matrix $\alpha_{3}$, where the generators react to each uncertain in-feed separately. The sum for all entries in each vertical column is one to ensure that the system is always balanced.

that $\boldsymbol{\alpha}_{3}$ takes on a block-like structure. For example, loads 5887 (located in zone 3 ) are covered mainly by generators 35 , 36 (connected at bus 77 and 80 in zone 3 ).

As discussed in the introduction, global balancing reduces the need for activation of balancing energy, while local balancing is more effective in reducing congestion. Policy III brings together the benefits in an optimal way by combining global and local balancing into "regional" balancing. On a regional level, imbalance netting is applied by choosing similar balancing vectors $\boldsymbol{\alpha}_{3(j, \text {.) }}$ for all $\omega_{j}$ in a given region (e.g., zone 3). However, the balancing vectors $\boldsymbol{\alpha}_{3(j, \cdot)}$ differ between regions that are separated by congestion, in order to reduce the uncertainty margins on the congested lines.

\section{CONCLusions AND Summary}

In this paper, we investigated different approaches to integrated balancing and congestion management in systems with significant levels of forecast uncertainty. We compared global balancing approaches (which only account for the total system deviation, indifferent of the location of the fluctuation) with a local balancing approach (where fluctuations are balanced based on their location in the system). While the global balancing policy requires less reserve activation, the local balancing policy can handle congestion more efficiently.

The approaches were quantitatively compared within a chance constrained OPF framework, which was extended to account for local balancing policies. In a case study based on the IEEE 118 bus system with 91 uncertain loads, we demonstrated that the local balancing policy reduces the cost of integrating wind power by reducing congestion due to forecast errors. Further, we observed that the optimization automatically chooses to perform imbalance netting in parts of the system without internal congestion.

In future work, we would like to account more accurately for cost of procuring and activating reserves, which has a significant influence on the benefit of local balancing (which requires more reserves). Further, we would like to extend the framework to consider zonal balancing, where fluctuations within a specified region are balanced together, as this might be a useful trade-off between computational complexity of the $\mathrm{CC}-\mathrm{OPF}$ and modelling of more flexible use of reserves.

\section{ACKNOWLEDGMENT}

We thank our partners within the UMBRELLA project, particularly members of the TSOs involved, for useful discussions leading to the ideas described in this paper.

\section{REFERENCES}

[1] Liu, Z. et al., "Challenges, experiences and possible solutions in transmission system operation with large wind integration," in 11th International Workshop on Large-Scale Integration of Wind Power into Power Systems, Lisbon, Portugal, 2012.

[2] "Supporting Document for the Network Code on LoadFrequency Control and Reserves," ENTSO-E, Tech. Rep., June 2013. [Online]. Available: https://www.entsoe.eu/fileadmin/user_upload/ library/resources/LCFR/130628-NC_LFCR-Issue1.pdf

[3] "Network Code on Load Frequency Control \& Reserves (NC LFCR)," ENTSO-E, Tech. Rep., June 2013. [Online]. Available: http://networkcodes.entsoe.eu/wp-content/uploads/2013/08/ 130628-NC_LFCR-Supporting_Document-Issue1.pdf

[4] "Press Release: International Grid Control Cooperation (IGCC) increases quality in operation and brings benefits on energy and monetary level," International Grid Control Cooperation, June 26 2014. [Online]. Available: https://www.transnetbw.de/de/presse/ presseinformationen/presseinformation?id=121

[5] Y. Gebrekiros, G. Doorman, S. Jaehnert, and H. Farahmand, "Balancing energy market integration considering grid constraints," in PowerTech, 2015 IEEE Eindhoven, June 2015, pp. 1-6.

[6] L. Roald, F. Oldewurtel, T. Krause, and G. Andersson, "Analytical reformulation of security constrained optimal power flow with probabilistic constraints," in IEEE PowerTech Conference, Grenoble, France, 2013.

[7] D. Bienstock, M. Chertkov, and S. Harnett, "Chance-constrained optimal power flow: Risk-aware network control under uncertainty," SIAM Review, vol. 56, no. 3, pp. 461-495, 2014.

[8] M. Vrakopoulou, K. Margellos, J. Lygeros, and G. Andersson, "Probabilistic guarantees for the N-1 security of systems with wind power generation," in PMAPS 2012, Istanbul, Turkey, 2012.

[9] — "A Probabilistic Framework for Reserve Scheduling and N-1 Security Assessment of Systems With High Wind Power Penetration," IEEE Trans. Power Systems, vol. 28, no. 4, pp. 3885-3896, 2013.

[10] G. Calafiore and M. C. Campi, "The scenario approach to robust control design," IEEE Trans. Automatic Control, vol. 51, pp. 742-753, 2006.

[11] S. Boyd and L. Vandenberghe, Convex Optimization. Cambridge, UK: Cambridge University Press, 2004.

[12] S. Dasgupta, D. Hsu, and N. Verma, "A concentration theorem for projections," in 22nd Conference on Uncertainty in Artificial Intelligence (UAI), 2006.

[13] B. Li and J. Mathieu, "Analytical reformulation of chance-constrained optimal power flow with uncertain load control," in PowerTech, 2015 IEEE Eindhoven, June 2015, pp. 1-6.

[14] "IEEE 118-bus, 54-unit, 24-hour system," Electrical and Computer Engineering Department, Illinois Institute of Technology, Tech. Rep. [Online]. Available: http://motor.ece.iit.edu/data/JEAS_IEEE118.doc

[15] J. Lofberg, "Yalmip : a toolbox for modeling and optimization in matlab," in Computer Aided Control Systems Design, 2004 IEEE International Symposium on, Sept 2004, pp. 284-289. 